OPEN ACCESS

Edited by:

Jinhui Wang

South China Normal University, China

Reviewed by:

Qinghua He,

Southwest University, China

Qiang Gao,

Sichuan University, China

*Correspondence:

Ti-Fei Yuan

ytf0707@126.com

Yulian Zhu

zyljully@163.com

tThese authors have contributed equally to this work and share first

authorship

Specialty section:

This article was submitted to Neural Technology, a section of the journal

Frontiers in Neuroscience

Received: 26 March 2020

Accepted: 11 May 2020

Published: 25 June 2020

Citation:

Yu F, Tang X, Hu R, Liang S,

Wang W, Tian S, Wu Y, Yuan T-F and

Zhu Y (2020) The After-Effect of Accelerated Intermittent Theta

Burst Stimulation at Different Session

Intervals. Front. Neurosci. 14:576.

doi: 10.3389/fnins.2020.00576

\section{The After-Effect of Accelerated Intermittent Theta Burst Stimulation at Different Session Intervals}

\author{
Fengyun $\mathrm{Yu}^{1,2+}$, Xinwei Tang ${ }^{1+}$, Ruiping Hu ${ }^{1+}$, Sijie Liang ${ }^{2}$, Weining Wang ${ }^{2}$, Shan Tian ${ }^{1}$, \\ Yi Wu ${ }^{1}$, Ti-Fei Yuan ${ }^{3,4 *}$ and Yulian Zhu ${ }^{1 *}$ \\ ${ }^{1}$ Department of Rehabilitation Medicine, Huashan Hospital, Fudan University, Shanghai, China, ${ }^{2}$ School of Kinesiology, \\ Shanghai University of Sport, Shanghai, China, ${ }^{3}$ Shanghai Key Laboratory of Psychotic Disorders, Shanghai Mental Health \\ Center, Shanghai Jiao Tong University School of Medicine, Shanghai, China, ${ }^{4}$ Co-innovation Center of Neuroregeneration, \\ Nantong University, Nantong, China
}

Objective: The study aims to investigate the after-effect of three sessions of intermittent theta-burst stimulation (iTBS) on motor cortical excitability. The iTBS was induced over the primary motor cortex (M1) at different time intervals.

Methods: The study has a crossover design. Sixteen participants were assigned to three groups and received different accelerated iTBS (aiTBS) protocols during each visit: (1) three continuous sessions with no interval (iTBS18000); (2) three iTBS sessions with 10-min intervals (iTBS600 $\times 3 * 10$ ); and (3) three iTBS sessions with 30-min intervals (iTBS600 $\times 3 * 30$ ). As washout period, each visit is separated by at least 7 days. We measured the motor cortical excitability changes and intracortical inhibition.

Results: A dose of 1,800 pulses of aiTBS per day is tolerable. The iTBS1800 led to a reduced cortical excitability; whereas iTBS600 $\times 3^{* 10}$ and iTBS600 $\times 3 * 30$ enhanced cortical excitability to a differential extent. After a total dose of 1,800 pulses, iTBS600 $\times 3 * 30$ exhibited the longer effect and highest percentage of individuals with enhanced cortical excitability.

Conclusion: The results suggest that aiTBS protocols at different time intervals result in different motor cortical excitability after-effects.

Keywords: theta burst stimulation, accelerated, motor cortex, cortical plasticity, stimulation interval

\section{HIGHLIGHTS}

- Accelerated TBS attracts attention in clinical settings.

- The study examined cortical plasticity induced by 3 sessions of iTBS at different intervals.

- Time interval affects cortical plasticity of accelerated TBS significantly.

\section{INTRODUCTION}

Transcranial magnetic stimulation (TMS) is a non-invasive neural regulation technique that can be used to modulate cortical excitability in the brain (Nordmann et al., 2015). Theta-burst stimulation (TBS) is a patterned repetitive paradigm that evokes cortical plasticity in a much shorter time period 
(Huang et al., 2005). Intermittent TBS (iTBS) can up-regulate cortical excitability and induces long-term potentiation (LTP). Conversely, continuous TBS (cTBS) down-regulates cortical excitability and induces long-term depression (LTD)-like effects (Wischnewski and Schutter, 2015). The after-effect of iTBS is time-varying; reportedly after a single iTBS, the maximum excitatory cortical effect is reached within $10 \mathrm{~min}$, and the excitatory effect gradually returns to the initial state after $30 \mathrm{~min}$ of iTBS (Wischnewski and Schutter, 2015; Chung et al., 2016).

Recently, it is proposed that accelerated iTBS (aiTBS), where repeated blocks of iTBS are applied, might bring improved clinically beneficial (Caeyenberghs et al., 2019). This might be due to its stronger induction effects on cortical plasticity. Interestingly, it is proposed that longer iTBS protocol with two blocks of 600 pulses iTBS does not elicit the similar cortical plasticity as single iTBS; for instance, the plasticity reverses (Gamboa et al., 2010). This result might be explained by metaplasticity effect - induction of new synaptic plasticity is dependent on historical synaptic activity (e.g., dosage, time interval of previous protocols) (Muller-Dahlhaus and Ziemann, 2015). However, few studies systematically examined the importance of time interval among different TBS sessions.

Studies have shown that the cortical excitatory effect of iTBS is dose-dependent, three-serially blocks of iTBS applied to the motor cortex; the effect of increasing cortical excitability was significantly higher than that of two blocks of iTBS (Nettekoven et al., 2014, 2015). However, previous studies have focused on exploring the effects of two repeated blocks of iTBS with different intervals or single iTBS (Gamboa et al., 2011; Chung et al., 2018; Tse et al., 2018); repeated triple blocks of iTBS studies are lacking.

Williams et al. (2018) first stated that 10 sessions of iTBS1800 per day are an effective treatment for refractory depression; this aiTBS protocol was then named Stanford Accelerated Intelligent Neuromodulation Therapy (SAINT) (Cole et al., 2019). The present study compared the effects of triple blocks of iTBS600 (total 1,800 pulses per session) at different time intervals ( 0 , 10 , and $30 \mathrm{~min}$ ) on motor cortical excitability in a crossover design. Our main purpose was to identify potentially optimized time intervals for aiTBS applications, in order to maximize the evoked cortical plasticity in terms of motor-evoked potentials (MEPs) amplitude, lasting effect, and minimum individual differences. The secondary purpose was to understand its mechanism by observing the tendency of iTBS1800 in regulating cortical excitability.

\section{MATERIALS AND METHODS}

\section{Participants}

Sixteen healthy male volunteers, aged from 20 to 30 years (mean and $\mathrm{SD}=22.75 \pm 2.62$ years), were recruited for the present study (Table 1). All participants were right-handed, as verified using the Edinburgh Handedness Inventory (Oldfield, 1971). None of the participants had any history of neurological or psychiatric disorders, serious illnesses, or epilepsy or took potentially hazardous drugs before the application of TMS. Participants provided informed consent prior to the experiment,
TABLE 1 | Basic information of subjects.

\begin{tabular}{|c|c|c|c|c|}
\hline Subjects & $\begin{array}{c}\text { Age } \\
\text { (years) }\end{array}$ & Gender & $\begin{array}{c}\text { Dominant } \\
\text { hemisphere }\end{array}$ & $\begin{array}{c}\text { Stimulation } \\
\text { site }\end{array}$ \\
\hline 1 & 21 & $\mathrm{M}$ & $L$ & LM1 \\
\hline 2 & 22 & $M$ & $\mathrm{~L}$ & LM1 \\
\hline 3 & 28 & $M$ & $\mathrm{~L}$ & LM1 \\
\hline 4 & 24 & $M$ & $\mathrm{~L}$ & LM1 \\
\hline 5 & 21 & $M$ & $\mathrm{~L}$ & LM1 \\
\hline 6 & 22 & M & $\mathrm{L}$ & LM1 \\
\hline 7 & 22 & $M$ & $L$ & LM1 \\
\hline 8 & 22 & $M$ & $\mathrm{~L}$ & LM1 \\
\hline 9 & 22 & M & $\mathrm{L}$ & LM1 \\
\hline 10 & 23 & $\mathrm{M}$ & $\mathrm{L}$ & LM1 \\
\hline 11 & 20 & $M$ & $\mathrm{~L}$ & LM1 \\
\hline 12 & 30 & M & $\mathrm{L}$ & LM1 \\
\hline 13 & 22 & $\mathrm{M}$ & $\mathrm{L}$ & LM1 \\
\hline 14 & 21 & $M$ & $\mathrm{~L}$ & LM1 \\
\hline 15 & 22 & M & $\mathrm{L}$ & LM1 \\
\hline 16 & 21 & $M$ & $\mathrm{~L}$ & LM1 \\
\hline Mean & 22.75 & & & \\
\hline SD & 2.62 & & & \\
\hline
\end{tabular}

and the experimental protocol was approved by Huashan Institutional Review Board.

\section{Experiment Design}

This study is a single-blind, crossover design. Each subject received iTBS protocols in three different regimens with randomized sequences: continuous 1,800 pulse iTBS stimulation (iTBS1800), three blocks of iTBS with 10-min interval (iTBS600 $\times 3^{*} 10$ ), and three blocks of iTBS with $30-\mathrm{min}$ interval (iTBS600 $\times 3^{*} 30$ ). Each session was 1 week apart to avoid potential lasting effects (Figure 1).

\section{TMS and EMG Procedure}

Subjects were advised to get enough sleep, avoid strenuous exercise, or drink stimulating beverages (e.g., coffee or tea) before taking part in the experiment. Before the first iTBS session, participants were told relevant experimental procedures; they underwent a thorough TMS safety screening procedure, and written informed consent was obtained. The experiment was conducted in a separate, quiet room. Participants were seated in a comfortable position on a recliner with armrests and were instructed to relax. The participants then wore a positioning scalp cap to reduce sliding between the hair and the stimulus coil. During the entire experiment, the environment was quiet, and the participants were not allowed to talk, sleep, or use their mobile phones.

Single TMS to the left primary motor cortex (LM1), which controls the right hand, was performed using an OSF-pTMS magnetic stimulator (O.SELF Company, Wuhan, China) with a figure-of-eight-shaped coil. The stimulating coil was held tangentially to the skull with the coil handle pointing backward and laterally $45^{\circ}$ away from the anterior-posterior axis. To assess 


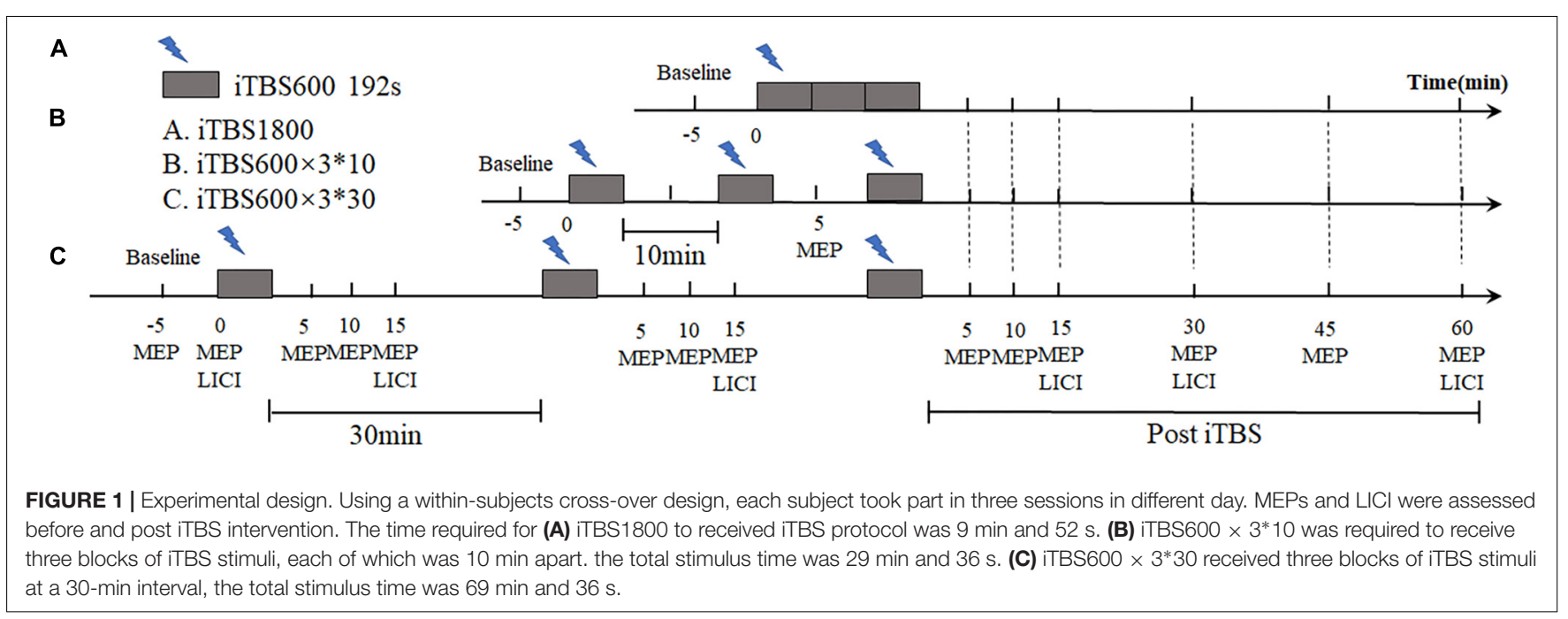

the motor cortex excitability, a surface electromyography (EMG) was recorded by attaching a pair of $\mathrm{Ag}-\mathrm{Ag} / \mathrm{Cl}$ electrodes to the first dorsal interosseous (FDI) muscle of the participants' right hand. Participants' muscle relaxation was observed by visual and EMG monitoring.

Cortical excitability was assessed by measuring the peak-topeak amplitude of MEPs from the non-dominant FDI at rest. The stimulation intensities (as a percentage of maximal stimulator output) of TMS were increased to evoke $\mathrm{MEP}_{\text {rightFDI }}$ wave 1$\mathrm{mV}$ peak-to-peak $\left(\mathrm{SI}_{1 \mathrm{mV}}\right)$; participants received 10 consecutive single pulses to the target point of the left $\mathrm{M} 1$ at an interval of 5 s. Furthermore, to measure MEPs, the intensity remained constant at baseline intensity throughout each experimental session. Resting motion threshold (RMT) was defined as the minimal stimulation intensity that could induce at least 5 of 10 consecutive trials in the FDI muscle with MEPs peak-topeak wave amplitude greater than $50 \mu \mathrm{V}$. Participants' RMT and MEPs were remeasured before each session. To ensure the intraindividual reliability of cortical excitability, we recorded 2 sessions of MEP measurements of each participant, with a 5-min interval at baseline. once the average amplitude difference between the two measurements was less than $20 \%$, the experiment could begin. If not, the baseline MEPs was measured until, for two consecutive times, the average amplitude difference of MEPs was less than $20 \%$.

For three different iTBS protocols, MEPs were recorded by 10 single pulses with $1-\mathrm{mV}$ stimulus intensities $\left(\mathrm{SI}_{1 \mathrm{mV}}\right)$. And it should be measured twice at baseline and at time points 5, 10, 15, 30,45 , and 60 min post-1,800 pulses. A total of eight measures of cortical excitability were required for iTBS1800. Furthermore, two sessions had break between interventions; MEPs were also measured at intervention intervals extra. The MEPs of iTBS600 $\times 3^{*} 10$ secession were recorded at $5 \mathrm{~min}$ when there was a break between interventions. A total of 10 measurements of cortical excitability were required for this protocol, and iTBS600 $\times 3^{*} 30$ session MEPs were recorded at 5, 10, and $15 \mathrm{~min}$ during intervention interval times. A total of 14 measurements of cortical excitability were required for this protocol.
Long interval intracortical inhibition (LICI) was assessed using a paired-pulse TMS protocol. In a very short period (100 or $150 \mathrm{~ms}$ ), two consecutive stimuli were performed in the same hemisphere. The intensity of both conditioning stimulus (CS) and test stimulus (TS) was above threshold. The conditioning pulse inhibited the original MEP. We tested LICI with a CS intensity set at $120 \%$ of RMT; the TS intensity was the same as the measured cortical excitability that could evoke an $\mathrm{MEP}_{\text {rightFDI }}$ of $1-\mathrm{mV}$ amplitude. The interstimulus interval was $150 \mathrm{~ms}$. Ten trials with single pulses (unconditioned) and 10 trials with paired pulses (conditioned) were recorded at an alternating order with an interval of $5 \mathrm{~s}$. The intensity of both conditional and stimulus pulses was above the threshold. LICI was measured at 15 and $60 \mathrm{~min}$ after each protocol of 1,800 pulses iTBS. For iTBS600 $\times 3^{*} 30$, LICI was also measured at time point of $15 \mathrm{~min}$ between the interval of two blocks of iTBS.

\section{Intermittent Theta-Burst Stimulation}

The iTBS pattern consisted of bursts containing three pulses at $50 \mathrm{~Hz}$ repeated at $5 \mathrm{~Hz} ; 2$-s train of TBS was repeated every $10 \mathrm{~s}$ for a total of $192 \mathrm{~s}$ (600 pulses) (Huang et al., 2005). The stimulation intensity of the experiment was set at $70 \%$ of RMT.

\section{Statistics Analysis}

SPSS version 22 (Statistical Package for the Social Sciences; IBM, Armonk, NY, United States) software was used for the statistical analysis of the data. To calculate plasticity, the MEPs were normalized to baseline MEP amplitude for each participant. LICI was expressed as the ratio of conditioned MEPs to unconditioned MEPs. All data (participant's age, RMT, $\mathrm{SI}_{1 \mathrm{mV}}$, MEP amplitude, MEP latency, and LICI) were expressed as mean \pm SD. One-way analysis of variance (ANOVA) was used to compare RMT, S1mV, MEP amplitude, and LICI at baseline between different sessions. Each time point after each iTBS protocol was compared with the baseline used paired $t$ test to analyze the after-effects of each iTBS conditions. To examine the effect of different iTBS intervals on cortical excitability and inhibition, repeated-measures ANOVAs (reANOVAs) were used to test the main effects of TREATMENT 
(iTBS1800, iTBS600 $\times 3^{*} 10$, iTBS600 $\times 3^{*} 30$ ) and TIME (baseline, 5, 10, 15, 30, 45, 60) on MEP amplitude and LICI. The sphericity was verified used the Mauchly test; when not met, Greenhouse-Geisser was used to correct for this. Pearson correlations were used to assess the relationship between the LTPlike plasticity induced by different TMS conditions, whereas the correlation was determined between LICI of baseline and mean MEP amplitude at 10, 30, and 60 min after iTBS. The correlation between the change of LICI and MEP amplitude at 15 and $60 \mathrm{~min}$ compared to baseline was also calculated by Pearson correlation test. Statistically significant values are defined as $P<0.05$.

\section{RESULTS}

All participants completed three sessions in the study, and no side effects were reported by the subjects during or after the experimental sessions. No significant differences were found when the ANOVAs were performed to test RMT $\left(F_{2,15}=0.01\right.$, $p=0.990)$, MEP amplitude $\left(F_{2,15}=1.27, p=0.290\right), \mathrm{SI}_{1 \mathrm{mv}}$ $\left(F_{2,15}=0.03, p=0.975\right)$, or LICI $\left(F_{2,15}=0.66, p=0.521\right)$ among the three visits (Table 2).

\section{Temporal Changes of Cortical Excitability in Different iTBS Protocol}

The duration of the after-effects of each individual iTBS protocol was examined by pairing $t$ test to baseline. Instead of inducing facilitation, the iTBS1800 suppressed MEP amplitude, which was significant at the 5 -min time point $(p=0.049)$. However, the MEP amplitude gradually recovered to its baseline, and facilitation occurred at 50 min post-1,800 pulses (Figure 2A).

For the iTBS600 $\times 3^{*} 10$ group, the facilitation effects on MEPs amplitude were significant at time point $30 \mathrm{~min}(p=0.015)$ from the baseline. The MEPs amplitude at time $5 \mathrm{~min}$ post-1,800 pulses was slightly larger than 5 min after 600 and 1,200 pulses stimulus, but statistically not significant $(p=0.914, p=0.728$, respectively) (Figure $2 \mathbf{B}$ ).

For the iTBS600 $\times 3^{*} 30$ group, the facilitation effect was still evident after $120 \mathrm{~min}$. In this iTBS paradigm, significant differences were seen if compared to the baseline at the time point $10 \mathrm{~min}(p=0.016)$ after 600 pulses; $10 \mathrm{~min}(p=0.012)$ and $15 \mathrm{~min}(p=0.019)$ after 1,200 pulses; and $5 \mathrm{~min}(p=0.013)$, $10 \min (p=0.018)$, and $60 \mathrm{~min}(p=0.030)$ after 1,800 pulses (Figure 2C).

TABLE 2 | Subjects' baseline RMT, MEP amplitude, SI $_{1 \mathrm{mv}}$, and LICI when started three different iTBS conditions.

\begin{tabular}{llllll}
\hline & $\begin{array}{l}\text { iTBS1800 } \\
(\mathbf{N}=\mathbf{1 6})\end{array}$ & $\begin{array}{l}\text { iTBS600 } \times \mathbf{3}^{*} \mathbf{1 0} \\
(\mathbf{N}=\mathbf{1 6})\end{array}$ & $\begin{array}{l}\text { iTBS600 } \times \mathbf{3}^{*} \mathbf{3 0} \\
(\mathbf{N}=\mathbf{1 6})\end{array}$ & $\boldsymbol{F}$ & $\boldsymbol{P}$ \\
\hline $\mathrm{RMT}$ & $39.69 \pm$ & $40.31 \pm 14.70$ & $40.38 \pm 14.45$ & 0.010 & 0.990 \\
$(\% \mathrm{MSO})$ & 15.99 & & & & \\
$\begin{array}{l}\mathrm{MEP} \\
(\mathrm{mV})\end{array}$ & $1.03 \pm 0.23$ & $1.22 \pm 0.43$ & $1.14 \pm 0.35$ & 1.272 & 0.290 \\
$\begin{array}{l}\mathrm{Sl} \mathrm{1mv}_{\mathrm{mv}} \\
(\% \mathrm{MSO})\end{array}$ & $50.8 \pm 20.05$ & $51.88 \pm 19.82$ & $52.25 \pm 17.19$ & 0.025 & 0.975 \\
$\mathrm{LICl}$ & $0.37 \pm 0.46$ & $0.22 \pm 0.35$ & $0.24 \pm 0.41$ & 0.662 & 0.521
\end{tabular}

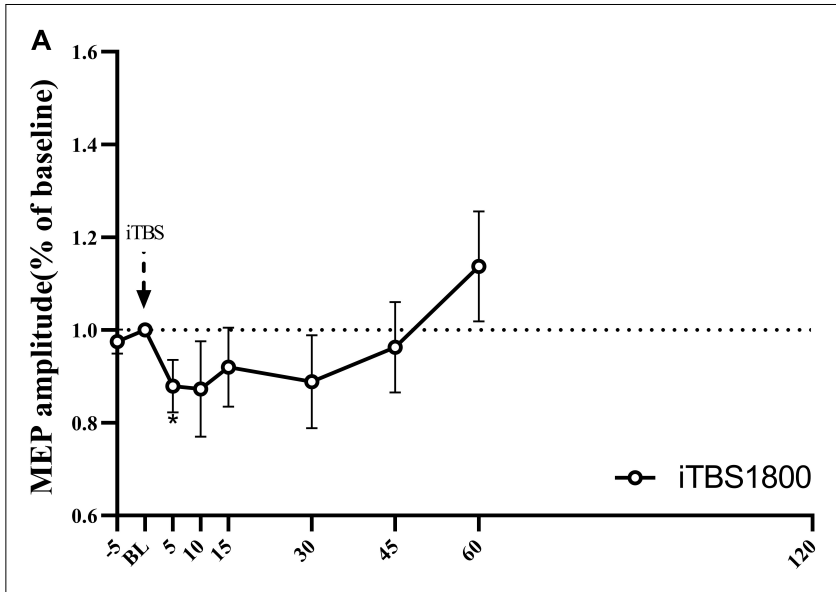

B

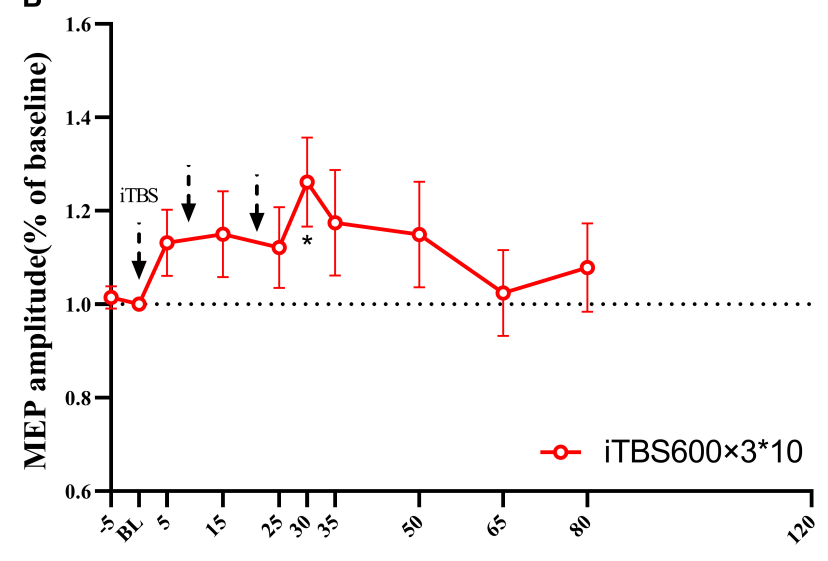

C

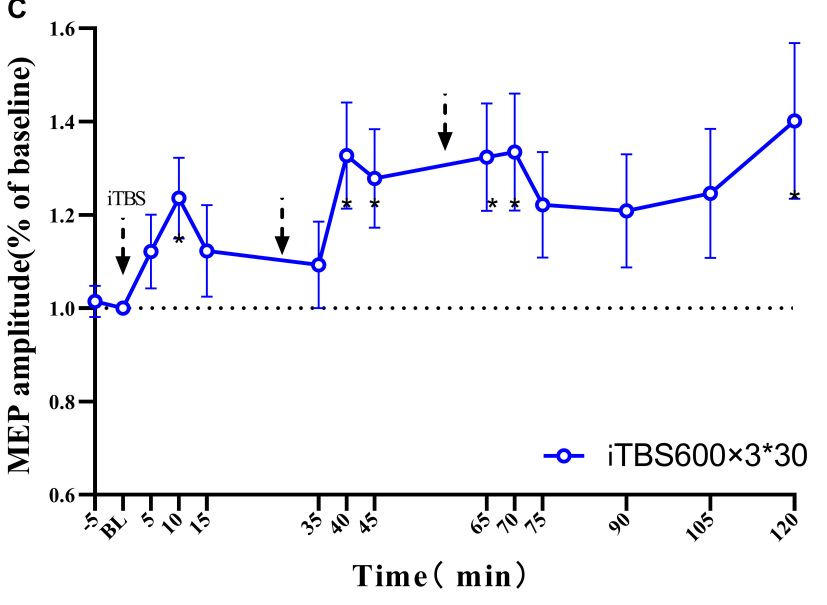

FIGURE 2 | MEPs amplitude at stimulation interval after each block of iTBS and up to 60 min post 1800 pulses of iTBS in difference iTBS conditions. For each cluster of (A) iTBS1800 (B) iTBS600 × 3*10 (C) iTBS600 × 3*30.

Asterisks indicate statistical significance between the MEPs amplitude at that time point and the baseline MEPs amplitude $\left({ }^{\star} P<0.05\right)$.

\section{After-Effect of 1,800-Pulse Dosage}

Two-way reANOVAs were employed to compare the differences across the three groups: MEP responses were aligned to the completion of 1,800 pulses (Table 3 ). The results revealed a significant main effect of the factors TREATMENT $\left(F_{2,30}=3.734\right.$, 
TABLE 3 | Normalized MEP amplitude for baseline and post stimulation measurement.

\begin{tabular}{|c|c|c|c|c|c|c|c|}
\hline & \multicolumn{7}{|c|}{ Time after stimulation (min) } \\
\hline & Baseline & T5 & T10 & T15 & T30 & T45 & T60 \\
\hline iTBS1800 $(N=16)$ & $1.00 \pm 0.00$ & $0.88 \pm 0.23$ & $0.87 \pm 0.41$ & $0.92 \pm 0.34$ & $0.89 \pm 0.40$ & $0.96 \pm 0.39$ & $1.14 \pm 0.48$ \\
\hline iTBS600 × 3*10 $(N=16)$ & $1.00 \pm 0.00$ & $1.12 \pm 0.35$ & $1.26 \pm 0.38$ & $1.17 \pm 0.45$ & $1.15 \pm 0.45$ & $1.02 \pm 0.37$ & $1.08 \pm 0.38$ \\
\hline iTBS600 × $3 * 30(N=16)$ & $1.00 \pm 0.00$ & $1.32 \pm 0.46$ & $1.33 \pm 0.50$ & $1.22 \pm 0.45$ & $1.21 \pm 0.48$ & $1.25 \pm 0.55$ & $1.40 \pm 0.67$ \\
\hline
\end{tabular}

$p=0.036)$, TIME $\left(F_{6,90}=2.886, p=0.013\right)$, and the interaction TREATMENT $\times \operatorname{TIME}\left(F_{12,180}=2.004, p=0.026\right)$. For the interaction, we analyzed the simple effect of TREATMENT and TIME separately by one-way reANOVAs. For the factors of TREATMENT, there were significant simple effect at $5 \mathrm{~min}$ $\left(F_{2,30}=7.423, p=0.002\right)$ and $10 \min \left(F_{2,30}=5.715, p=0.008\right)$ post-1,800 pulse iTBS; for the factor of TIME, significance was found only in iTBS600 $\times 3^{*} 30$ group $\left(F_{6,90}=2.609, p=0.022\right)$.

According to the ANOVAs results, there is a significant difference in TREATMENT at the time points $5 \mathrm{~min}$ $(p=0.004)$ and $10 \mathrm{~min}(p=0.009)$. Post hoc with Bonferroni correction revealed significant differences between iTBS1800 and iTBS600 $\times 3^{*} 30$ at $5 \mathrm{~min}(p=0.003)$ and $10 \mathrm{~min}(p=0.013)$ and significant differences between iTBS1800 and iTBS600 $\times 3^{*} 10$ at $10 \min (p=0.045)$ (Figure 3).

\section{Individual Exhibits Distinct Plasticity Responses to Different Protocols}

We checked if one individual exhibited similar levels of plasticity to the three types of TBS protocols. Hence, the Pearson correlation test was used to assess the correlation among the three sessions. No correlation for cortical plasticity was identified between the three sessions (Figure 4).

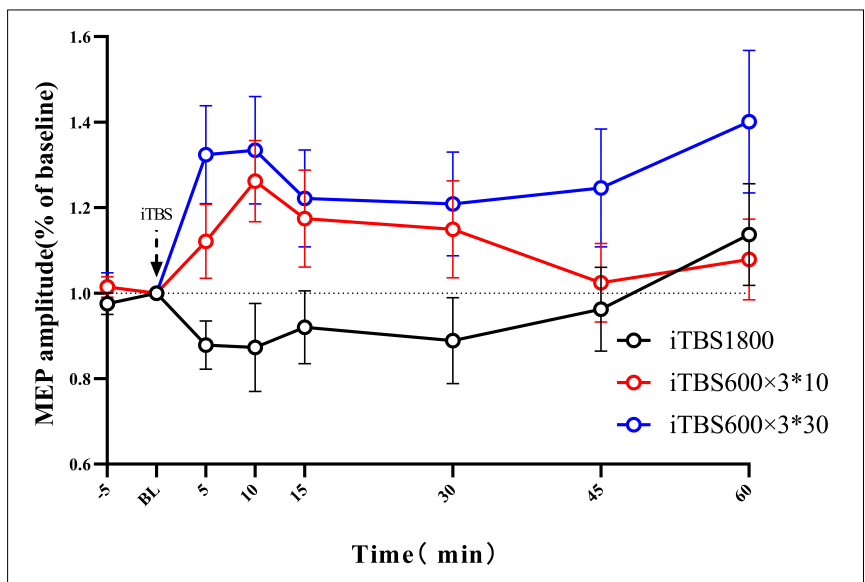

FIGURE 3 | The after-effect of 1800 pulses iTBS on MEP amplitude at times $5,10,15,30,45$, and 60 min following three different iTBS condition. MEPs amplitude were normalized to baseline.

\section{Effect of iTBS on Intracortical Inhibition}

We used reANOVAs to see if different iTBS conditions affected the inhibitory intracortical interneuronal circuitry. The reANOVAs for LICI, at each time point in the experiment, showed no effects correlated to the TREATMENT $\left(F_{2,30}=1.530, p=0.233\right)$, TIME $\left(F_{2,30}=2.135, p=0.136\right)$, or TREATMENT $\times \operatorname{TIME}\left(F_{4,60}=1.157, p=0.332\right)$ interaction.

\section{Interindividual and Intraindividual Effects}

The individual responses to the three different protocols were plotted (Figure 5). In addition, when the multiple time point average value was $>1.2$, it was considered as facilitation responder; when multiple time point average value was less than 0.8 , it was considered as inhibitory responders; those who compared the change in MEP to baseline between 1.2 and 0.8 were considered no responders. We took the response of the subjects to the first $30 \mathrm{~min}$ in iTBS600 $\times 3^{*} 30$ group as a classic iTBS response, and the percentage of subjects showing a facilitation was 50\% (Figure 6A). In the iTBS1800, 25\% of subjects showed facilitation, whereas $62.5 \%$ showed inhibition (Figure 6B). In the iTBS600 $\times 3^{*} 10,56.25 \%$ of subjects showed facilitation, and $12.3 \%$ were inhibited, respectively (Figure 6C). The facilitation effect of iTBS600 $\times 3^{*} 30$ was more obvious than the other two iTBS conditions; $75 \%$ of subjects showed facilitation, and 6.25\% were inhibited (Figure 6D).

\section{DISCUSSION}

This study is an exploratory study to identify a clinically effective protocol for aiTBS and understand the effect and mechanism of iTBS1800 on cortical excitability. We reported that the dosedependent accumulative effect for cortical plasticity is affected by the interval from each iTBS session. Considering the potential side effects induced from total dosage, the 30-min interval three blocks of iTB600 protocol exhibited the longest potentiation effects on the motor cortex. There was no significant inhibitory effect on cortical excitability in iTBS1800, but 50 min after 1,800 pulses of iTBS, the facilitation effect gradually appears.

This is the first study where continuous 1,800 pulses of iTBS to $\mathrm{M} 1$ are performed. Within $50 \mathrm{~min}$ of the stimulus, the aftereffects of iTBS1800 were the same as previous studies that performed iTBS1200 (Gamboa et al., 2010) and cTBS (Guerra et al., 2019), which inhibits cortical excitability. Because we did 


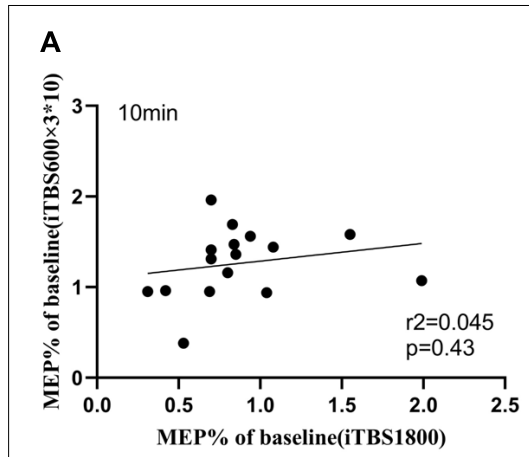

D

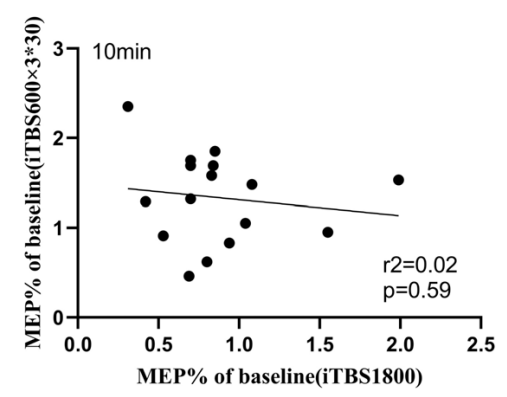

G

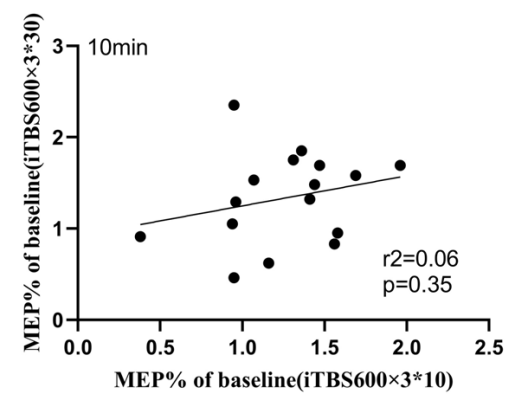

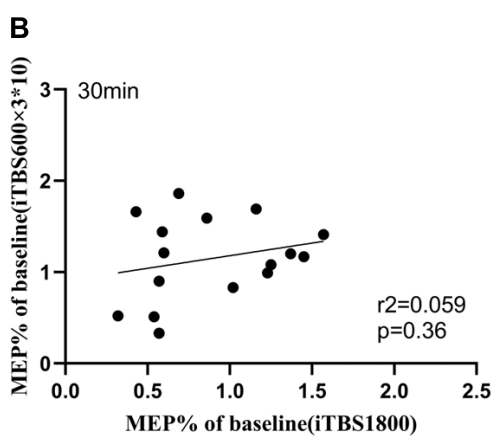

E

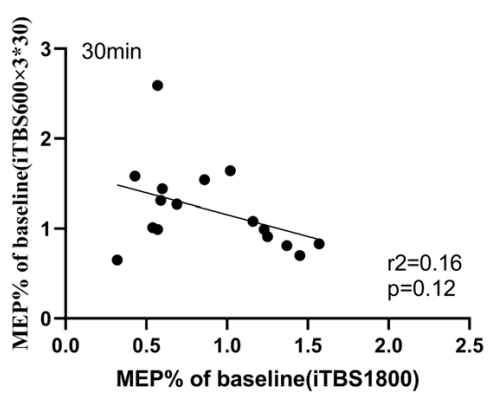

H

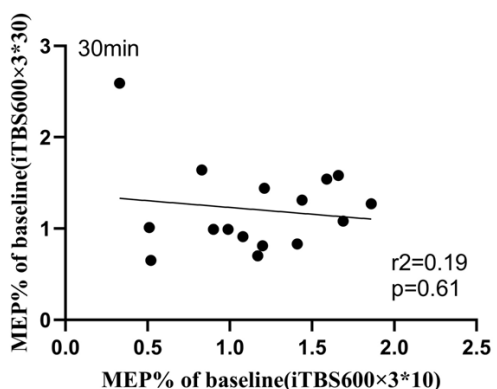

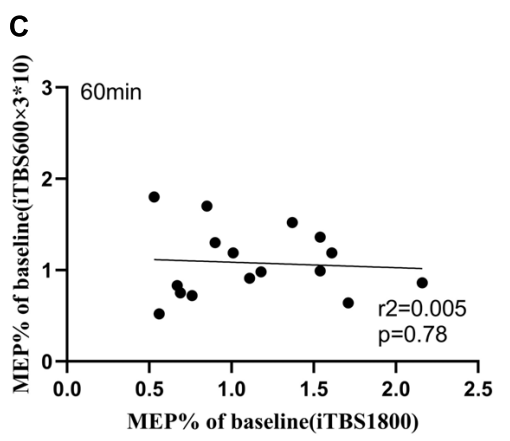

$\mathbf{F}$

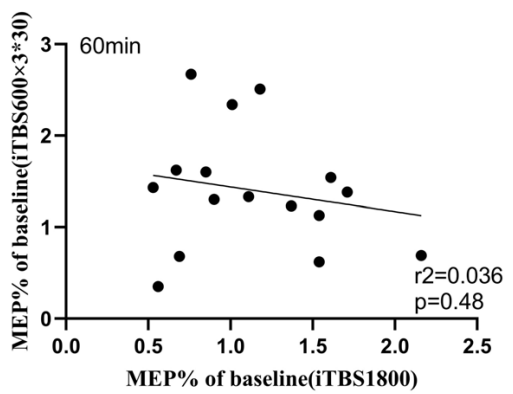

I

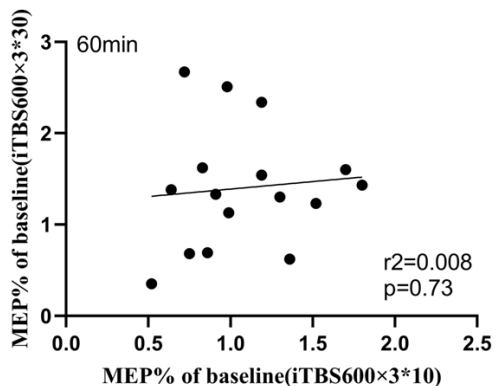

FIGURE 4 | Relationship between iTBS1800, iTBS600 $\times 3^{*} 10$, and iTBS600 $\times 3^{*} 30$ at time points 10, 30, and 60 min. Correlation between the normalized grand average MEPs amplitude following different iTBS conditions (A) MEPs amplitude correlation of iTBS1800 and iTBS600 $\times 3^{*} 10$ at 10 min (B) MEPs amplitude correlation of iTBS1800 and iTBS600 × 3*10 at $30 \mathrm{~min}$ (C) MEPs amplitude correlation of iTBS1800 and iTBS600 $\times 3^{*} 10$ at 60 min (D) MEPs amplitude correlation of iTBS1800 and iTBS600 $\times 3^{*} 30$ at $10 \mathrm{~min}$ (E) MEPs amplitude correlation of iTBS1800 and iTBS600 $\times 3^{*} 30$ at $30 \mathrm{~min}$ (F) MEPs amplitude correlation of iTBS1800 and iTBS600 $\times 3^{*} 30$ at $60 \mathrm{~min}$ (G) MEPs amplitude correlation of iTBS600 $\times 3^{*} 10$ and iTBS600 $\times 3^{*} 30$ at 10 min (H) MEPs amplitude correlation of iTBS600 × 3*10 and iTBS600 × 3*30 at $30 \mathrm{~min}$ (I) MEPs amplitude correlation of iTBS600 $\times$ 3*10 and iTBS600 $\times$ 3*30 at $60 \mathrm{~min}$.
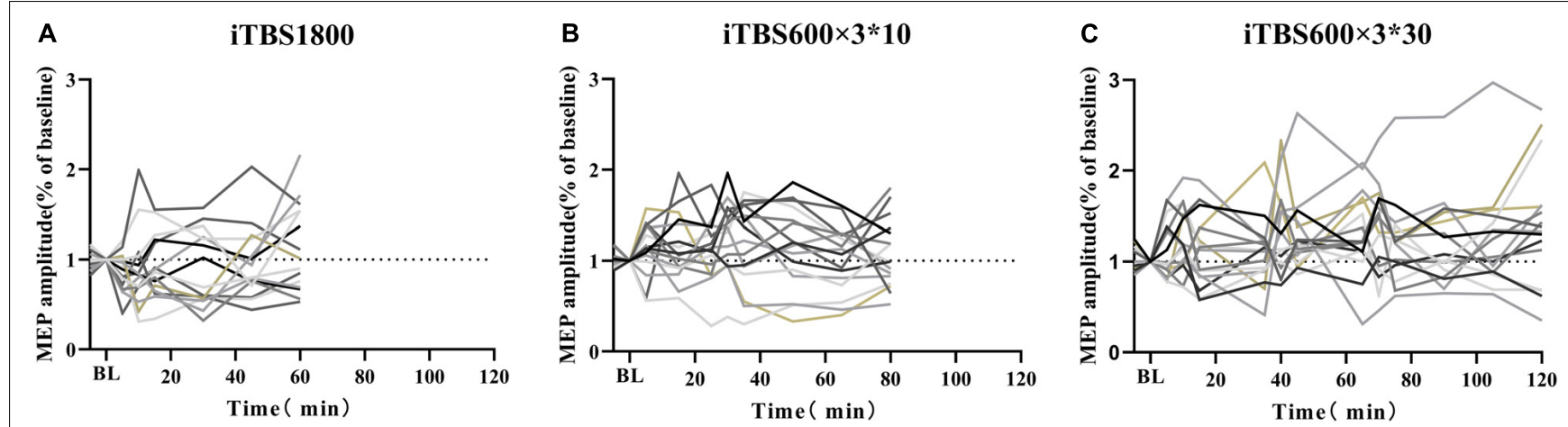

FIGURE 5 | Individual response to iTBS after 1800 pulses stimulation in three different intervals. (A) iTBS1800 (B) iTBS600 $\times 3 * 10$ (C) iTBS600 $\times 3 * 30$. 

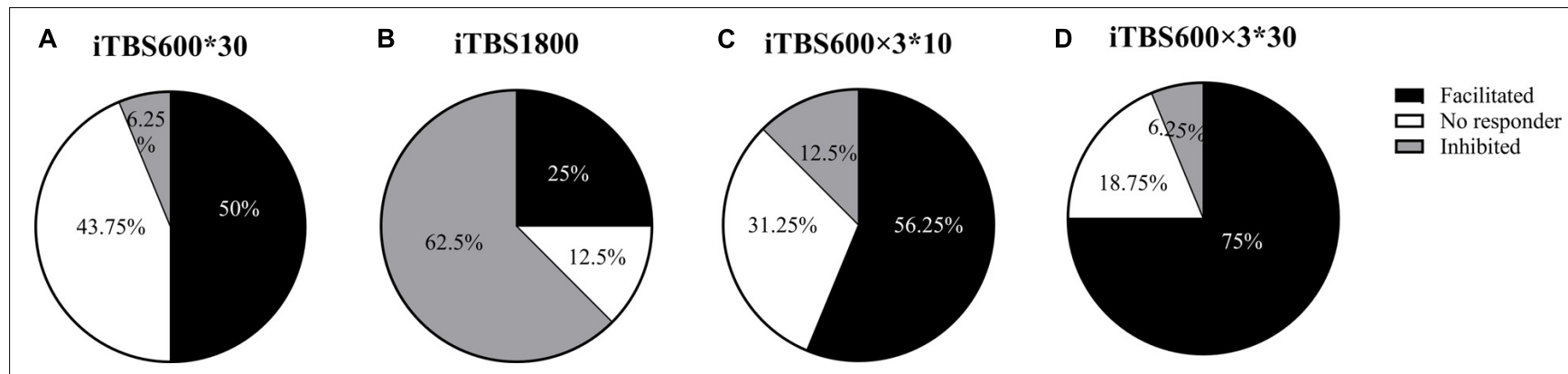

FIGURE 6 | The percentage of subjects that responded to different iTBS conditions. (A) one blocks of iTBS (B) iTBS1800 (C) iTBS600 $\times 3 * 10$ (D) iTBS600 $\times 3 * 30$.

not compare the iTBS1200 group and classical iTBS group, we could not compare the inhibitory effect of the three protocols. However, contrary to our findings, another study that prolonged the theta burst stimulation to 1,200 pulses (Hsu et al., 2011) showed that iTBS1200 enhanced facilitation and time course of corticospinal tract. The different results may be due to the different stimulus dose and because we conducted iTBS1800 immediately after the RMT was measured, whereas in the experiment of Hsu et al. (2011) there was a 5-min rest after RMT was measured. The excitatory effect begins after $50 \mathrm{~min}$ of stimulation. Because our experiment was not observed for a longer time, the reasons behind the change in amplitude are unclear. This is consistent with other studies where previously saturated LTP was significantly enhanced by several multiblocks of iTBS to the cerebral cortex if the interval was more than 50 min (Kramár et al., 2012; Thomson et al., 2019). Our research could explain the mechanism by which the SAINT protocol [10 sessions of iTBS1800 with interval of $50 \mathrm{~min}$ to the left dorsolateral prefrontal cortex (DLPFC)] effectively treats refractory depression. Although the cortical areas we stimulated were different from those used to treat depression, the effect of iTBS1800 on cortical excitability is consistent.

For classical iTBS, the MEP amplitude is maximized at 10-15 min post-iTBS, and excitation gradually decreased (Wischnewski and Schutter, 2015). In our study, we have chosen the time interval of 10 and $30 \mathrm{~min}$, compared to iTBS1800 and classical iTBS. We found that repeated blocks of iTBS did have a cumulative effect when the appropriate intervals were selected. Previous studies demonstrated that cortical plasticity evoked by 1,200 pulses of iTBS did not mimic a single session of iTBS (600 pulses), depending on the time interval between the 2 iTBS sessions. No and 5-min intervals led to depression (Gamboa et al., 2011; Tse et al., 2018), whereas 15-min interval led to a potentiation similar to as a single session (Nettekoven et al., 2014). In addition, 15-min interval of three sessions of iTBS induced enhanced cortical plasticity than a single session of iTBS (Nettekoven et al., 2014), which is consistent in our results. All these results suggested that an aiTBS sessions should have an interval larger than $15 \mathrm{~min}$ to prevent depression-like plasticity responses.

The superposition and reversal of the after-effect of repeated blocks of iTBS may be explained by the following hypothesis. The effects of TBS are dependent on $N$-methyl-D-aspartate receptors (Huang et al., 2007), which allows calcium ions to enter the postsynaptic membrane and trigger LTP or LTD (Citri and Malenka, 2008). This influx triggers the generation of LTP, and the increased concentration of calcium ions in the postsynaptic membrane is one of the necessary conditions for the formation of LTP (Cho et al., 2001; Abraham, 2008). Conversely, both LTP and LTD are triggered by the same second messenger $\mathrm{Ca}^{2+}$. There is a calcium level called "no man's land" between the LTP and LTD $\mathrm{Ca}^{2+}$ zones that results in no plasticity. Whether LTP or LTD occurs depends on the $\mathrm{Ca}^{2+}$ levels after TBS protocol (Lisman, 2001). Conversely, a theory of synaptic metaplasticity was proposed by Fung and Robinson (2014). They simulated the oscillations of intracellular calcium, calcium conductance, and plasticity signals. The results showed that the relationship between the after-effect with stimulus dose and time of iTBS presented waveforms; inhibition and facilitation alternate with the change of stimulus dose and intervention time.

Intracortical inhibition (ICI) and intracortical facilitation (ICF) measurements are commonly used to explore intracortical inhibition and facilitatory circuits. Short interval intracortical inhibition (SICI) and LICI were mediated via postsynaptic $\gamma$-aminobutyric acid (GABA) A and B receptors, respectively; LICI leads to a long-lasting inhibitory postsynaptic potential and described as late cortical disinhibition (Werhahn et al., 1999; Cash et al., 2010). Previous studies suggested that SICI significantly increased or reduced following iTBS and cTBS, whereas ICF did not change after both TBS protocols (Huang et al., 2005; Murakami et al., 2008). According to a meta-analysis, SICI changes only occur in a very short time point ( $<5 \mathrm{~min})$ after cTBS; no significant differences were found in SICI at any time point with iTBS (Chung et al., 2016). There were no changes in SICI following classical iTBS and break repeat blocks of iTBS (Tse et al., 2018). In our study, no changes in LICI were found after iTBS intervention or at different intervals of iTBS conditions.

Previous studies also reported high intersubject variability in cortical plasticity studies. The subjects were given the same iTBS protocols; $43 \%$ of the subjects were responders that increase the MEPs amplitude, whereas 57\% were non-responders (Lopez-Alonso et al., 2014). Furthermore, the same subject exhibited different after-effects to iTBS on their different visits 
(Schilberg et al., 2017). One study confirmed a relationship between the dose of stimulation and individual responses to TMS (Fitzgerald et al., 2020). The response to repeated iTBS varies depending on the individual (Tse et al., 2018). In our current study, only $25 \%$ of subjects showed facilitation in TBS1800, but an increase to $56.25 \%$ of subjects showed facilitation in iTBS600 $\times 3^{*} 10$. The facilitation effect of iTBS600 $\times 3^{*} 30$ was more obvious than the other two iTBS conditions where $75 \%$ of subjects showed facilitation. The results suggest that an individual's response to TBS could also be modulated by prior TBS exposures. Furthermore, there is no correlation between the after-effect of each iTBS condition at any time point, indicating that the mechanism of different schemes may be different, and the response of different individuals to different iTBS schemes may be different.

There are several limitations in our study. First, we measured cortical excitability and cortical inhibition only within $60 \mathrm{~min}$ of iTBS protocols. It is unclear what happens after $60 \mathrm{~min}$; for iTBS1800, the cortical excitability after $60 \mathrm{~min}$ shifted toward greater facilitation or a return to the original state. Second, we tested only the direct effect of aiTBS on M1 and recruited only young males in order to reduce the interindividual variability. Therefore, more studies are needed to confirm the effect of aiTBS on different brain regions (e.g., DLPFC, cerebellum, and Broca) and other healthy subjects, as well as patients.

In conclusion, the varied time intervals of iTBS sessions contributed to distinct cortical plasticity responses in aiTBS protocols. Our data indicate that three blocks of iTBS with a 30min interval induce prominent cortical plasticity. We suggest that it might be necessary to test patients' response to iTBS protocols in order to improve clinical efficacy.

\section{DATA AVAILABILITY STATEMENT}

The original contributions presented in the study are included in the article/supplementary material, further inquiries can be directed to the corresponding authors.

\section{REFERENCES}

Abraham, W. C. (2008). Metaplasticity: tuning synapses and networks for plasticity. Nat. Rev. Neurosci. 9:387. doi: 10.1038/nrn2356

Caeyenberghs, K., Duprat, R., Leemans, A., Hosseini, H., Wilson, P. H., Klooster, D., et al. (2019). Accelerated intermittent theta burst stimulation in major depression induces decreases in modularity: a connectome analysis. Netw. Neurosci. 3, 157-172. doi: 10.1162/netn_a_00060

Cash, R. F., Ziemann, U., Murray, K., and Thickbroom, G. W. (2010). Late cortical disinhibition in human motor cortex: a triple-pulse transcranial magnetic stimulation study. J. Neurophysiol. 103, 511-518. doi: 10.1152/jn.00782.2009

Cho, K., Aggleton, J. P., Brown, M. W., and Bashir, Z. (2001). An experimental test of the role of postsynaptic calcium levels in determining synaptic strength using perirhinal cortex of rat. J. Physiol. 532(Pt 2), 459-466. doi: 10.1111/j.1469-7793. 2001.0459f.x

Chung, S. W., Hill, A. T., Rogasch, N. C., Hoy, K. E., and Fitzgerald, P. B. (2016). Use of theta-burst stimulation in changing excitability of motor cortex: a systematic review and meta-analysis. Neurosci. Biobehav. Rev. 63, 43-64. doi: 10.1016/j.neubiorev.2016.01.008

\section{ETHICS STATEMENT}

The studies involving human participants were reviewed and approved by the Huashan Hospital Affiliated to Fudan University Institutional Review Board (HIRB). The patients/participants provided their written informed consent to participate in this study.

\section{AUTHOR CONTRIBUTIONS}

FY: conceptualization, methodology, validation, formal analysis, investigation, data curation, writing - original draft, and visualization. XT: software and investigation. $\mathrm{RH}$ : resources, supervision, and funding acquisition. SL, WW, and ST: investigation. YW: project administration. T-FY: conceptualization, methodology, writing - review and editing, and supervision. YZ: resources, supervision, funding acquisition, writing - review and editing, project administration, and supervision. All authors contributed to the article and approved the submitted version.

\section{FUNDING}

This work was supported by the Key Projects of Shanghai Science and Technology on Biomedicine (no. 18411962300), Shanghai Health and Family Planning Commission project (no. 201840225), and Shanghai Municipal Key Clinical Specialty (no. shslczdzk02702).

\section{ACKNOWLEDGMENTS}

The authors are thankful for all the subjects' voluntary contributions during the completion of this study and special thanks to Chong Xi and Li Pan for their help in the experiment.

Chung, S. W., Rogasch, N. C., Hoy, K. E., and Fitzgerald, P. B. (2018). The effect of single and repeated prefrontal intermittent theta burst stimulation on cortical reactivity and working memory. Brain Stimul. 11, 566-574. doi: 10.1016/j.brs. 2018.01.002

Citri, A., and Malenka, R. C. (2008). Synaptic plasticity: multiple forms, functions, and mechanisms. Neuropsychopharmacology 33, 18-41. doi: 10.1038/sj.npp. 1301559

Cole, E. J., Stimpson, K. H., Bentzley, B. S., Gulser, M., Cherian, K., Tischler, C., et al. (2019). Stanford accelerated intelligent neuromodulation therapy for treatment-resistant depression (SAINT-TRD). Brain Stimul. 12:402. doi: 10. 1016/j.brs.2018.12.299

Fitzgerald, P. B., Hoy, K. E., Reynolds, J., Singh, A., Gunewardene, R., Slack, C., et al. (2020). A pragmatic randomized controlled trial exploring the relationship between pulse number and response to repetitive transcranial magnetic stimulation treatment in depression. Brain Stimul. 13, 145-152. doi: 10.1016/j.brs.2019.09.001

Fung, P. K., and Robinson, P. A. (2014). Neural field theory of synaptic metaplasticity with applications to theta burst stimulation. J. Theor. Biol. 340, 164-176. doi: 10.1016/j.jtbi.2013.09.021 
Gamboa, O. L., Antal, A., Laczo, B., Moliadze, V., Nitsche, M. A., and Paulus, W. (2011). Impact of repetitive theta burst stimulation on motor cortex excitability. Brain Stimul. 4, 145-151. doi: 10.1016/j.brs.2010.09.008

Gamboa, O. L., Antal, A., Moliadze, V., and Paulus, W. (2010). Simply longer is not better: reversal of theta burst after-effect with prolonged stimulation. Exp. Brain Res. 204, 181-187. doi: 10.1007/s00221-010-2293-4

Guerra, A., Suppa, A., Asci, F., De Marco, G., D’Onofrio, V., Bologna, M., et al. (2019). LTD-like plasticity of the human primary motor cortex can be reversed by gamma-tACS. Brain Stimul. 12, 1490-1499. doi: 10.1016/j.brs.2019.06.029

Hsu, Y. F., Liao, K. K., Lee, P. L., Tsai, Y. A., Yeh, C. L., Lai, K. L., et al. (2011). Intermittent theta burst stimulation over primary motor cortex enhances movement-related beta synchronisation. Clin. Neurophysiol. 122, 2260-2267. doi: 10.1016/j.clinph.2011.03.027

Huang, Y. Z., Chen, R. S., Rothwell, J. C., and Wen, H. Y. (2007). The aftereffect of human theta burst stimulation is NMDA receptor dependent. Clin. Neurophysiol. 118, 1028-1032. doi: 10.1016/j.clinph.2007.01.021

Huang, Y. Z., Edwards, M. J., Rounis, E., Bhatia, K. P., and Rothwell, J. C. (2005). Theta burst stimulation of the human motor cortex. Neuron 45, 201-206. doi: 10.1016/j.neuron.2004.12.033

Kramár, E. A., Babayan, A. H., Gavin, C. F., Cox, C. D., Jafari, M., Gall, C. M., et al. (2012). Synaptic evidence for the efficacy of spaced learning. Proc. Natl. Acad. Sci. U.S.A. 109, 5121-26. doi: 10.1073/pnas.1120700109

Lisman, J. E. (2001). Three Ca 2+ levels affect plasticity differently: the LTP zone, the LTD zone and no man's land. J. Physiol. 532, 285-285. doi: 10.1111/j.14697793.2001.0285f.x

Lopez-Alonso, V., Cheeran, B., Rio-Rodriguez, D., and Fernandez-Del-Olmo, M. (2014). Inter-individual variability in response to non-invasive brain stimulation paradigms. Brain Stimul. 7, 372-380. doi: 10.1016/j.brs.2014.02.004

Muller-Dahlhaus, F., and Ziemann, U. (2015). Metaplasticity in human cortex. Neuroscientist 21, 185-202. doi: 10.1177/1073858414526645

Murakami, T., Sakuma, K., Nomura, T., Nakashima, K., and Hashimoto, I. (2008). High-frequency oscillations change in parallel with short-interval intracortical inhibition after theta burst magnetic stimulation. Clin. Neurophysiol. 119, 301-308. doi: 10.1016/j.clinph.2007.10.012

Nettekoven, C., Volz, L. J., Kutscha, M., Pool, E. M., Rehme, A. K., Eickhoff, S. B., et al. (2014). Dose-dependent effects of theta burst rTMS on cortical excitability and resting-state connectivity of the human motor system. J. Neurosci. 34, 6849-6859. doi: 10.1523/JNEUROSCI.4993-13.2014

Nettekoven, C., Volz, L. J., Leimbach, M., Pool, E. M., Rehme, A. K., Eickhoff, S. B., et al. (2015). Inter-individual variability in cortical excitability and motor network connectivity following multiple blocks of rTMS. Neuroimage 118, 209-218. doi: 10.1016/j.neuroimage.2015.06.004
Nordmann, G., Azorina, V., Langguth, B., and Schecklmann, M. (2015). A systematic review of non-motor rTMS induced motor cortex plasticity. Front. Hum. Neurosci. 9:416. doi: 10.3389/fnhum.2015. 00416

Oldfield, R. C. (1971). The assessment and analysis of handedness: the Edinburgh inventory. Neuropsychologia 9, 97-113. doi: 10.1016/0028-3932(71) 90067-4

Schilberg, L., Schuhmann, T., and Sack, A. T. (2017). Interindividual variability and intraindividual reliability of intermittent theta burst stimulation-induced neuroplasticity mechanisms in the healthy brain. J. Cogn. Neurosci. 29, 10221032. doi: 10.1162/jocn_a_01100

Thomson, A. C., de Graaf, T. A., Kenis, G., Rutten, B. P. F., Schuhmann, T., and Sack, A. T. (2019). No additive meta plasticity effects of accelerated iTBS with short inter-session intervals. Brain Stimul. 12, 1301-1303. doi: 10.1016/j.brs. 2019.05.012

Tse, N. Y., Goldsworthy, M. R., Ridding, M. C., Coxon, J. P., Fitzgerald, P. B., Fornito, A., et al. (2018). The effect of stimulation interval on plasticity following repeated blocks of intermittent theta burst stimulation. Sci. Rep. 8:8526. doi: 10.1038/s41598-018-26791-w

Werhahn, K. J., Kunesch, E., Noachtar, S., Benecke, R., and Classen, J. (1999). Differential effects on motorcortical inhibition induced by blockade of GABA uptake in humans. J. Physiol. 517, 591-597. doi: 10.1111/j.1469-7793.1999. 0591t.x

Williams, N. R., Sudheimer, K. D., Bentzley, B. S., Pannu, J., Stimpson, K. H., Duvio, D., et al. (2018). High-dose spaced theta-burst TMS as a rapid-acting antidepressant in highly refractory depression. Brain 141:e18. doi: 10.1093/ brain/awx379

Wischnewski, M., and Schutter, D. J. (2015). Efficacy and time course of theta burst stimulation in healthy humans. Brain Stimul. 8, 685-692. doi: 10.1016/j.brs. 2015.03.004

Conflict of Interest: The authors declare that the research was conducted in the absence of any commercial or financial relationships that could be construed as a potential conflict of interest.

Copyright (c) 2020 Yu, Tang, Hu, Liang, Wang, Tian, Wu, Yuan and Zhu. This is an open-access article distributed under the terms of the Creative Commons Attribution License (CC BY). The use, distribution or reproduction in other forums is permitted, provided the original author(s) and the copyright owner(s) are credited and that the original publication in this journal is cited, in accordance with accepted academic practice. No use, distribution or reproduction is permitted which does not comply with these terms. 